\title{
Disociación escafosemilunar. Resultado radiográfico de pacientes tratados con anclas
}

\author{
Scapholunate ligament injuries. Radiographic \\ results in patients treated with bone anchor
}

\author{
Natalia Delia Domínguez Chacón, ${ }^{*}$ José Antonio Rivas Montero, ${ }^{\ddagger}$ Alejandro Espinoza Gutiérrez§
}

\section{RESUMEN}

La inestabilidad escafosemilunar es la causa más frecuente de inestabilidad carpal. La reparación o reconstrucción del ligamento escafosemilunar está enfocada en eliminar los síntomas, estabilizar la articulación escafosemilunar y evitar la progresión o degeneración radiocarpal. El objetivo de este trabajo es evaluar los resultados radiográficos obtenidos en 11 pacientes operados con minianclas Mitek por diagnóstico de inestabilidad escafosemilunar. Se comparó esta medición en el postoperatorio inmediato para comprobar si el cierre del intervalo permanece. Material y métodos: Se analizaron los expedientes radiográficos de los pacientes con diagnóstico de disociación escafosemilunar tratados con anclas durante el año 2017 en el Instituto Nacional de Rehabilitación; se realizó una medición del intervalo en las radiografías de control a los dos, seis y 12 meses de seguimiento. Resultados: En este estudio se comprobó que, a pesar de la colocación de anclas, el cierre del intervalo no permanece intacto después de un año de postoperatorio. Discusión: En esta medición radiográfica se comprobó que el intervalo escafosemilunar tratado con anclas tiene tendencia hacia una reapertura al paso de los meses; sin embargo, ésta no llega a una nueva disociación y, según lo reportado en la literatura, tampoco tiene alguna repercusión clínica. Conclusiones: El tratamiento con anclas de la disociación escafosemilunar no permanece intacto después de un año de postoperatorio, aunque la reapertura del espacio no fue significativo, ni llega a parámetros de una nueva disociación en el promedio de los casos.

Palabras clave: Disociación, ligamento escafo-semilunar, anclas. Nivel de evidencia: III

\begin{abstract}
Scapholunate instability is the most common form of carpal instability. Repair or reconstruction of the scapholunate interosseus ligament is advocated to reduce symptoms, stabilize the scapholunate joint and avoid the progression of carpal degeneration. Aim of this study is to evaluate the radiographic results in 11 patients treated for scapholunate instability with minianchor Mitek. Material and methods: We analized the files of every patient with a scapholunate instability during 2017 in the National Rehabilitation Institute and measure the scaphoid lunate space in the immediate postoperative, after two, six, and 12 months to check if the clausure remains. Results: This study probed that the closure of this space does not remain after 1 year postoperatory, however this does affect clinically o became a new instability in most of cases. Discussion: This radiographic result probed that the scapholunate space clausure does not remain intact after one year, however this result doesn't became a new instability and according to literature does not have a clinical outcome. Conclusions: The treatment of closing the scapholunate space with anchors does not remain after 1 year postoperative, however it doesnt became a new instability or affect the patient clinicly according to literature.
\end{abstract}

Keywords: Scapholunate, ligament, bone anchor, wrist arthroscopy. Level of evidence: III
* Médico Especialista en Cirugía de Mano. Adscrito al Centro Médico ABC.

₹ Médico Especialista en Cirugía de Mano. Adscrito al Instituto Nacional de Rehabilitación.

$\S$ Jefe del Servicio de Mano y Microcirugía del Instituto Nacional de Rehabilitación.
Correspondencia: Natalia Delia Domínguez Chacón

Centro Médico ABC Santa Fe. Prolongación Vasco de Quiroga Núm. 4001,

Torre Santa Fe Hills, consultorio 701, Col. Santa Fe, 05348, Alcaldía Cuajimalpa, CDMX. Tel: 9154 3107; E-mail: dranataliadmgz@gmail.com

Abreviaturas:

$E S=$ Escafosemilunar.

INR = Instituto Nacional de Rehabilitación. 


\section{INTRODUCCIÓN}

Las lesiones de ligamentos carpales y la inestabilidad de los huesos carpales son lesiones complejas; con frecuencia se presentan en gente joven, deportista o en edad laboral. Estas lesiones pueden ser aisladas en pacientes con un único evento traumático como caídas o presentarse por traumatismos repetidos. Son difíciles de diagnosticar en su etapa aguda, ya que la radiografía simple no mostrará ningún cambio en etapas tempranas.

La inestabilidad escafosemilunar (ES) es la alteración biomecánica que resulta de la ruptura del ligamento interóseo entre los huesos escafoides y el semilunar, ligamento ES. La definición actual se ha extendido para incluir la disfunción sintomática e incluye la incapacidad de tolerar carga con una cinemática alterada de la fila proximal del carpo. ${ }^{1}$ Esta anomalía fue descrita por primera vez por Linscheid y Dobyns en 1972; es la causa más frecuente de inestabilidad carpal y se puede asociar a otras lesiones agudas o crónicas como fracturas de radio, fracturas de escafoides o pseudoartrosis de escafoides. ${ }^{2}$

El ligamento intrínseco ES se divide en tres regiones anatómicas: dorsal, proximal y palmar. La región dorsal es más gruesa y está compuesta de fibras cortas de colágeno orientadas transversalmente. ${ }^{3}$ Aunque el ligamento ES es el estabilizador primario de la articulación ES, puede aparecer una disfunción progresiva como resultado de la lesión de los estabilizadores secundarios. Los estabilizadores secundarios son: el ligamento radiocarpiano dorsal, el ligamento intercarpiano dorsal, el ligamento radio-escafo-hueso grande y el ligamento escafo-trapecio-trapezoidal. La mala alineación resultante (flexión y pronación del escafoides, así como extensión y supinación del semilunar) puede ser permanente, lo que determina una inestabilidad ES estática. ${ }^{4}$

La etiología más común es traumática: caída con extensión de muñeca, desviación cubital y supinación carpal, aunque también puede relacionarse a otras patologías como artritis reumatoide o resección quirúrgica excesiva de cápsula dorsal al extirpar gangliones. La correcta clasificación de la lesión puede ayudar en la selección del tratamiento adecuado. Existen varias opciones: fijación percutánea con clavos, reparación directa, capsulodesis, reconstrucción con injerto tendinoso además de las técnicas artroscópicas. En la inestabilidad dinámica reductible y sin cambios degenerativos está indicada la reparación artroscópica ${ }^{5}$ modificada con anclas. Ésta permite una reparación con reforzamiento sobre el ligamento dorsal intercarpal, lo que reconstruye el complejo dorsal ES. Además, se puede proteger con la colocación de un clavo de Kirschner por cuatro a seis semanas o con inmovilización. La reparación o reconstrucción del ligamento ES está enfocada en eliminar los síntomas, estabilizar la articulación ES y evitar la progresión o degeneración radiocarpal.

Aunque no existe un consenso actual sobre el mejor tratamiento de estas lesiones, el tratamiento más utilizado por ahora en etapas tempranas sin signos de artrosis es la reparación de ligamento con la colocación de dos minianclas metálicas, una en escafoides y otra en semilunar, para realizar una reparación del ligamento ES dorsal.

El tiempo que transcurre entre la lesión y el diagnóstico es esencial para elegir el tratamiento más adecuado, ya que está muy relacionado con la eficacia biomecánica de los ligamentos estabilizadores secundarios, puesto que éstos degeneran muy rápido (aproximadamente a las dos semanas), así que una correcta reinserción o reparación tiene mejor pronóstico entre más rápido se realice. ${ }^{6}$

El objetivo de este trabajo es evaluar el resultado radiográfico obtenido en pacientes tratados quirúrgicamente con minianclas metálicas Mitek para corregir la disociación ES en el postoperatorio inmediato, a los dos, seis y 12 meses, para comprobar si el cierre del intervalo ES permanece sin cambios.

\section{MATERIAL Y MÉTODOS}

Expediente radiográfico de pacientes con diagnóstico de disociación ES tratado con anclas en el Instituto Nacional de Rehabilitación (INR) durante 2017.

\section{RESULTADOS}

Se valoró radiográfica y retrospectivamente a 11 pacientes con diagnóstico de lesión ES, tratados quirúrgicamente durante 2017, en el Servicio de Mano y Microcirugía del INR.

Se capturó la información de la medición radiográfica en una tabla de Excel creada para el protocolo y se analizaron las siguientes variables.

De los 11 pacientes se encontró un rango de edad de 16 a 49 años, con un promedio de 33, lo cual coincide con que es una patología que predomina en gente joven. El género que prevaleció fue el masculino, sólo cuatro son mujeres, lo que concuerda con la literatura, siendo una lesión que se presenta más en los varones. 


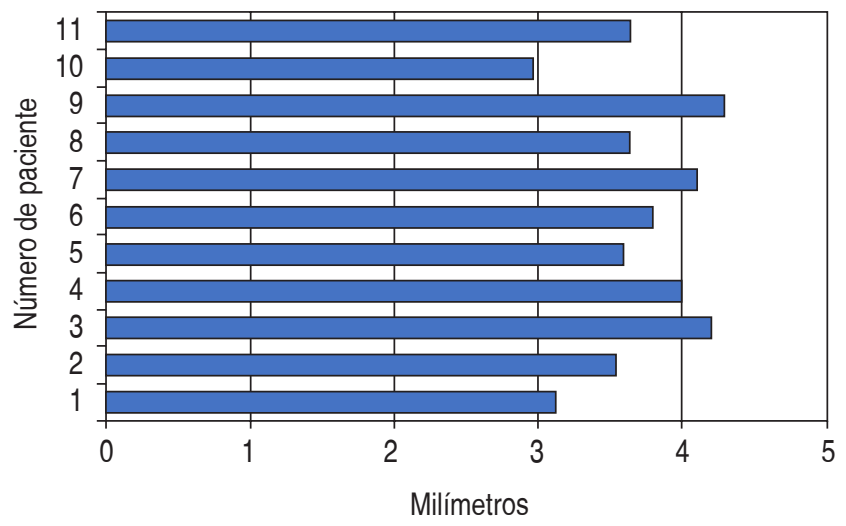

Figura 1: Medición prequirúrgica del espacio escafosemilunar en milímetros.

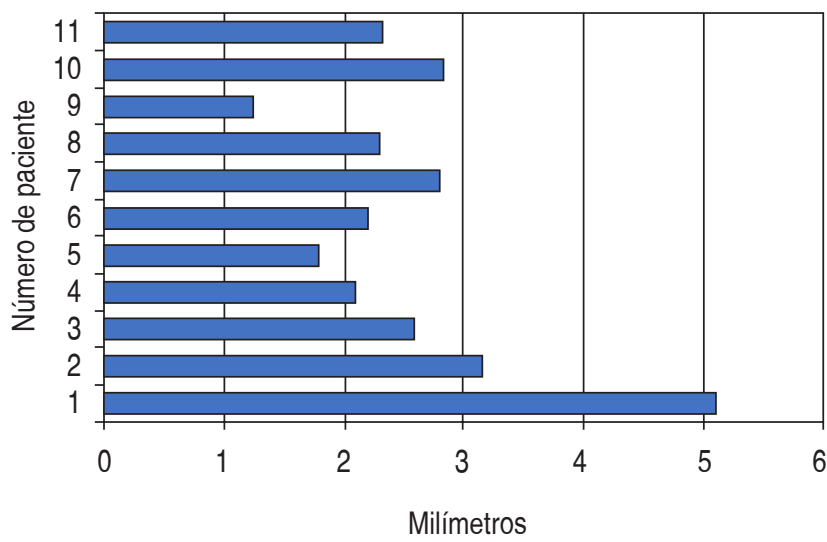

Figura 2: Medición postquirúrgica inmediata del espacio escafosemilunar en milímetros.

Medición radiográfica. Se obtuvo un promedio de $3.7 \mathrm{~mm}$ de apertura del espacio escafosemilunar prequirúrgica y un promedio de ES postquirúrgico de $2.5 \mathrm{~mm}$, lo cual traduce un cierre del intervalo de $1.2 \mathrm{~mm}$ en el postoperatorio inmediato tratado con anclas. Sin embargo, se observa una reapertura de 1 $\mathrm{mm}$ promedio a los dos meses, que permanece a los seis meses y de $2 \mathrm{~mm}$ a los 12 meses; no obstante, no llega a ser significativa, ya que el promedio después de un año permaneció en $2.9 \mathrm{~mm}$ (Figuras 1 a 5 ).

\section{DISCUSIÓN}

Se han descrito muchos procedimientos quirúrgicos para reparar la ruptura traumática del ligamento escafosemilunar, con el objetivo de mejorar el dolor de la muñeca y limitación funcional que provoca y, en especial, para prevenir o retrasar el proceso degenerativo que provoca.
Históricamente se intentó reparar sólo la porción volar del ligamento escafosemilunar o reparar ambos componentes (dorsal y volar) mediante un doble abordaje. ${ }^{5,7}$ Los estudios biomecánicos muestran que para lograr una cinemática carpal normal sólo es necesario reparar la porción dorsal del ligamento ES. ${ }^{8}$

Como sugiere Taleisnik, ${ }^{9}$ la mejor reparación del ligamento ES se realiza mediante un abordaje dorsal que nos permite una mejor exposición y una correcta reinserción ósea del ligamento; el uso de minianclas metálicas para corregir además la estabilidad rotatoria se ha descrito desde $1995 .^{10,11}$

Una revisión que se realizó de esta técnica por Minami y Kaneda demostró que esta técnica tuvo una reducción significativa de la inestabilidad ES postraumática y mantuvo rangos de movilidad de la muñeca aceptables. ${ }^{12}$ Bickert y su equipo repor-

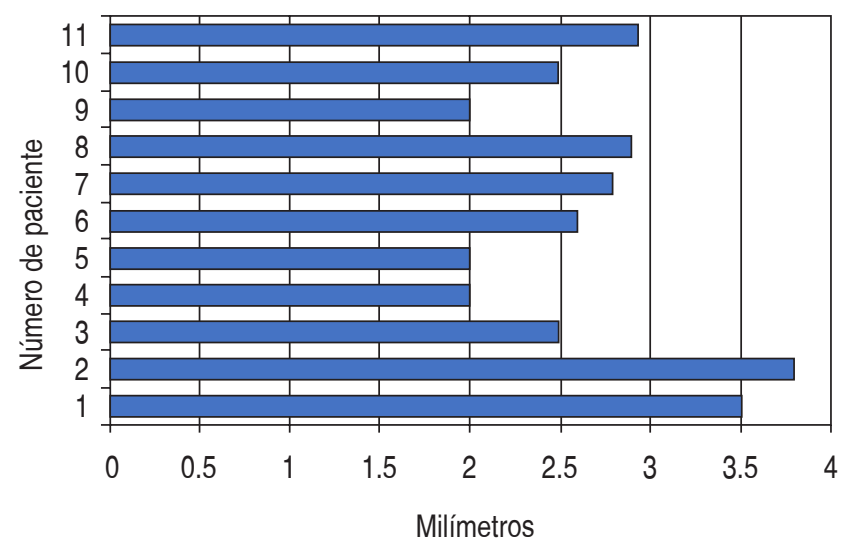

Figura 3: Medición a los dos meses postquirúrgicos del espacio escafosemilunar en milímetros.

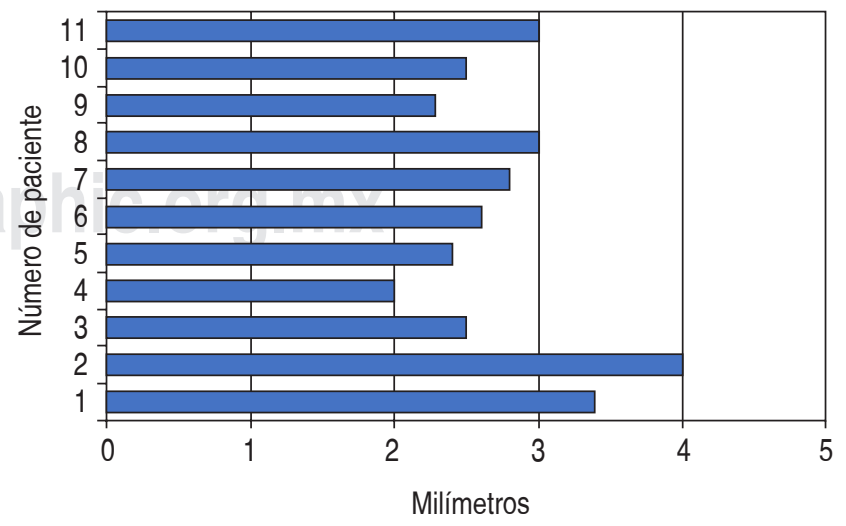

Figura 4: Medición a los seis meses postquirúrgicos del espacio escafosemilunar en milímetros. 


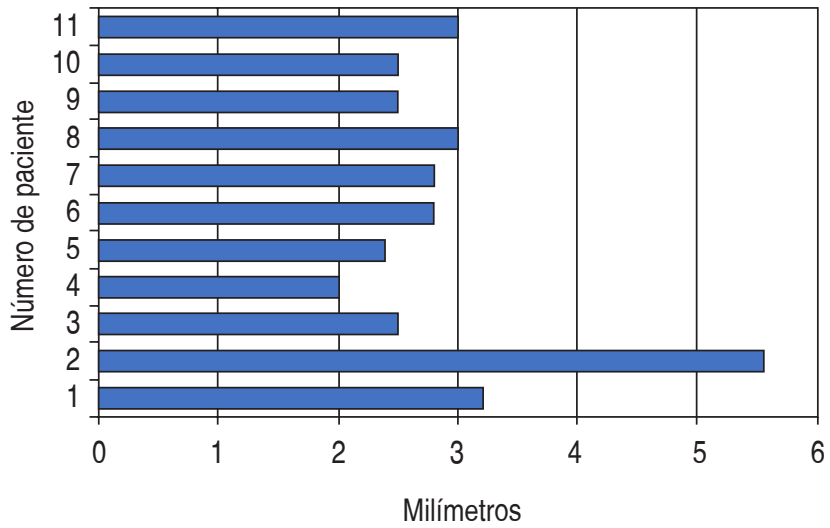

Figura 5: Medición a los 12 meses postquirúrgicos del espacio escafosemilunar en milímetros.

taron buenos resultados funcionales y radiográficos en una serie de pacientes con disociación ES tratados con anclas metálicas. ${ }^{13}$ Mientras Pilný reportó 94\% de excelentes resultados en 17 pacientes con ruptura aguda de ligamento ES, manejados con minianclas en un seguimiento a dos años. ${ }^{14}$

La mayoría de las fallas en esta técnica se debe a la fuerza del hueso grande sobre el espacio escafosemilunar que puede provocar una ruptura del ligamento reparado. ${ }^{15}$

\section{CONCLUSIÓN}

La reparación directa del ligamento ES usando anclas es generalmente exitosa para recuperar la estabilidad, con buenos resultados funcionales a corto y mediano plazo. La reparación abierta con abordaje dorsal permite una excelente visualización de la superficie articular del escafoides y semilunar para la correcta colocación de las anclas.

La medición radiográfica de este trabajo comprueba que, aunque el cierre inicial del intervalo obtenido en el postoperatorio inmediato no permanece después de un año, la reapertura del intervalo no es significativa ni llega a parámetros de una nueva disociación. Se ha comprobado que la falta de cierre del espacio ES no tiene repercusiones clínicas mientras no existan otras alteraciones mecánicas.
Consideramos que es necesario una correlación clínica de estos casos, ya que la reapertura del intervalo ES puede ser asintomática, además de un seguimiento a largo plazo, para descubrir si existe una reapertura o si en verdad el tratamiento en etapas tempranas detiene la historia natural de la enfermedad hacia alteraciones cinemáticas del carpo.

\section{BIBLIOGRAFÍA}

1. Green D. Green's operative surgery of the hand. 5th edition. New York, USA: Elsevier; 2007.

2. Kuo C, Wolfe S. Scapholunate instability: current concepts in diagnosis and management. J Hand Surg Am. 2008; 33 (6): 998-1013.

3. Moran SL, Cooney WP, Berger RA, Strickland J. Capsulodesis for the treatment of chronic scapholunate instability. J Hand Surg Am. 2005; 30 (1): 16-23.

4. Garcia-Elias M, Lluch AL, Stanley JK. Three-ligament tenodesis for the treatment of scapholunate dissociation: indications and surgical technique. J Hand Surg Am. 2006; 31 (1): 125-134.

5. Slater RR Jr, Szabo RM, Bay BK, Laubach J. Dorsal intercarpal ligament capsulodesis for scapholunate dissociation: biomechanical analysis in a cadaver model. J Hand Surg Am. 1999; 24 (2): 232-239.

6. Wahegaonkar AL, Mathoulin CL. Arthroscopic dorsal capsuloligamentous repair in the treatment of chronic scapho-lunate ligament tears. J Wrist Surg. 2013; 2 (2): 141-148.

7. Darlis NA, Weiser RW, Sotereanos DG. Partial scapholunate ligament injuries treated with arthroscopic debridement and thermal shrinkage. J Hand Surg Am. 2005; 30 (5): 908-914.

8. Cooney WP, Dobyns JH, Linscheid RL. Arthroscopy of the wrist: anatomy and classification of carpal instability. Arthroscopy. 1990; 6 (2): 133-140.

9. Taleisnik J. Post-traumatic carpal instability. Clin Orthop Relat Res. 1980; (149): 73-82.

10. Buch BD, Innis P, McClinton MA, Kotani Y. The Mitek Mini G2 suture anchor: biomechanical analysis of use in the hand. J Hand Surg Am. 1995; 20 (5): 877-881.

11. Cohen MS, Taleisnik J. Direct ligamentous repair of scapholunate dissociation with capsulodesis augmentation. Tech Hand Up Extrem Surg. 1998; 2 (1): 18-24.

12. Minami A, Kaneda K. Repair and/or reconstruction of scapholunate interosseous ligament in lunate and perilunate dislocations. J Hand Surg Am. 1993; 18 (6): 1099-1106.

13. Bickert B, Sauerbier M, Germann G. Scapholunate ligament repair using the Mitek bone anchor. J Hand Surg Br. 2000; 25 (2): 188-192.

14. Pilný J, Kubes J, Cizmár I, Visna P. Our experience with repair of the scapholunate ligament using the MITEK bone anchor. Acta Chir Orthop Traumatol Cech. 2005; 72 (6): 381-386.

15. Wyrick JD, Youse BD, Kiefhaber TR. Scapholunate ligament repair and capsulodesis for the treatment of static scapholunate dissociation. J Hand Surg Br. 1998; 23 (6): 776-780. 